\title{
Quantum phase space points for Wigner functions in finite-dimensional spaces
}

\author{
Alfredo Luis* \\ Departamento de Óptica, Facultad de Ciencias Físicas, Universidad Complutense, 28040 Madrid, Spain
}

(Received 6 December 2003; published 20 May 2004)

\begin{abstract}
We introduce quantum states associated with single phase space points in the Wigner formalism for finitedimensional spaces. We consider both continuous and discrete Wigner functions. This analysis provides a procedure for a direct practical observation of the Wigner functions for states and transformations without inversion formulas.
\end{abstract}

DOI: 10.1103/PhysRevA.69.052112

PACS number(s): 03.65.Ca, 42.50.Dv

\section{INTRODUCTION}

In recent times there has been an increasing interest in the practical determination of states and transformations [1,2]. Most of these practical procedures are based on the representation of quantum objects by functions on the classical phase space of the problem, specially the Wigner function [3-12].

Despite its classical resemblance, it is known that the Wigner function can take negative values, so this is not a true probability distribution and its practical determination requires more or less indirect procedures. Among them, the ones that require less inversion formulas are based on the fact that the Wigner function can be measured as an expectation value for each point in phase space [13]. In this regard, it is also worth pointing out that the square of the Wigner function is positive, so it can be measured as a probability distribution. This can be accomplished in quantum optics by mixing at a beam splitter the field state under investigation with its complex conjugate in the quadrature representation and measuring suitable quadratures of the output fields [14].

The occurrence of negative values of the Wigner function can be charged to a single fact: within the Wigner-Weyl operator-function correspondence there is no quantum state corresponding to a single point of the phase space. Given the relevance of the Wigner function we examine this issue further looking for quantum states as close as possible to be the quantum counterpart of a point of the classical phase space. Among other consequences this provides practical procedures approaching a direct observation of the Wigner function.

In Sec. II we recall basic formulas related to the Wigner function for states and transformations. In Sec. III we examine the quantum operators corresponding to phase space points and their representation by quantum states. In Sec. IV we apply this approach to the most relevant examples of Wigner function for finite-dimensional spaces.

\section{WIGNER FUNCTION FOR STATES AND TRANSFORMATIONS}

The Wigner function $W(\Omega)$ for a state $\rho$ can be defined as $[3-10]$

\footnotetext{
*Electronic address: alluis@ fis.ucm.es
}

$$
W(\Omega)=\operatorname{tr}[\rho \Delta(\Omega)],
$$

where $\rho$ is the density matrix representing the quantum state in the Hilbert space, the parameter $\Omega$ designates the points of the associated classical phase space, and $\Delta(\Omega)$ is a family of operators (phase space point operators). The inverse of the above correspondence is of the form

$$
\rho=\int d \Omega W(\Omega) \Delta(\Omega),
$$

for a suitable measure in phase space $d \Omega$.

Similarly, we can assign a Wigner function $\mathcal{U}\left(\Omega^{\prime}, \Omega\right)$ to an input-output transformation $[11,12]$

$$
\rho_{\text {out }}=\sum_{k} U_{k} \rho_{\text {in }} U_{k}^{\dagger},
$$

relating the Wigner functions $W_{\text {out }}\left(\Omega^{\prime}\right), W_{\text {in }}(\Omega)$ associated with the output and input states $\rho_{\text {out }}, \rho_{\text {in }}$, respectively,

$$
W_{\text {out }}\left(\Omega^{\prime}\right)=\int d \Omega \mathcal{U}\left(\Omega^{\prime}, \Omega\right) W_{\text {in }}(\Omega),
$$

being

$$
\mathcal{U}\left(\Omega^{\prime}, \Omega\right)=\sum_{k} \operatorname{tr}\left[\Delta\left(\Omega^{\prime}\right) U_{k} \Delta(\Omega) U_{k}^{\dagger}\right]
$$

Formulas for the inverse of Eq. (2.5) are also available but will not be necessary for this work [15].

\section{PHASE SPACE POINT STATES}

From the above expressions we can infer that $\Delta(\Omega)$ is the quantum operator associated with the classical phase space point $\Omega$ in the Wigner formalism. While classically a single point in phase space is a legitimate state, its operator counterpart $\Delta(\Omega)$ is not in general a quantum state. This is because in general $\Delta(\Omega)$ is not positive $[\Delta(\Omega)<0]$, and is not normalized $[\operatorname{tr} \Delta(\Omega) \neq 1]$. Positiveness and normalization are two necessary conditions for an operator to be a density matrix, according to the statistical interpretation of the quantum theory.

In order to look for a suitable connection between the phase space point operators and quantum states we may diagonalize $\Delta(\Omega)$ for each $\Omega$ 


$$
\Delta(\Omega)=\sum_{\ell} \lambda_{\ell}(\Omega)|\Omega, \ell\rangle\langle\Omega, \ell|,
$$

where $|\Omega, \ell\rangle$ are the eigenvectors and $\lambda_{\ell}(\Omega)$ the eigenvalues. Among other applications, this decomposition provides practical procedures to determine $W(\Omega)$ and $\mathcal{U}\left(\Omega^{\prime}, \Omega\right)$ based on the generation and detection of the states $|\Omega, \ell\rangle$, since they can be expressed as

$$
\begin{gathered}
W(\Omega)=\sum_{\ell} \lambda_{\ell}(\Omega)\langle\Omega, \ell|\rho| \Omega, \ell\rangle, \\
\mathcal{U}\left(\Omega^{\prime}, \Omega\right)=\sum_{k, \ell, m} \lambda_{\ell}(\Omega) \lambda_{m}\left(\Omega^{\prime}\right)\left|\left\langle\Omega^{\prime}, m\left|U_{k}\right| \Omega, \ell\right\rangle\right|^{2} .
\end{gathered}
$$

This strategy has been already proposed and carried out experimentally for a single mode of the electromagnetic field, being $|\Omega, \ell\rangle$ displaced number states [13]. Note that the lack of positiveness of the Wigner function is reflected on the fact that $\lambda_{\ell}(\Omega)$ can be negative [16].

The above decomposition (3.1) would also serve to determine quantum states closer to $\Delta(\Omega)$ as the eigenvectors with the maximum eigenvalue, for example. This idea will be pursued elsewhere. In this work we pretend to go a step further looking for states $\rho_{\Omega}$ embodying all the operator $\Delta(\Omega)$, instead of being determined only by part of its spectrum.

For infinite-dimensional Hilbert spaces it seems that there is no way of avoiding the two difficulties $\Delta(\Omega)<0$ and $\operatorname{tr} \Delta(\Omega) \neq 1$ simultaneously, so we will focus on finite dimensional spaces. In such a case the two difficulties can be solved at once in the form

$$
\rho_{\Omega}=\frac{1}{N \lambda(\Omega)+\eta(\Omega) \operatorname{tr} \Delta(\Omega)}\left[\eta(\Omega) \Delta(\Omega)+\lambda(\Omega) I_{N}\right],
$$

where $N$ is the dimension of the Hilbert space, $\eta(\Omega)$ is a real scalar, $I_{N}$ is the identity, and $\lambda(\Omega) \geqslant-\lambda_{\min }(\Omega)$, being $\lambda_{\min }(\Omega)$ the minimum eigenvalue of $\eta(\Omega) \Delta(\Omega)$. It is clear that $\rho_{\Omega}$ is always a legitimate density matrix $\rho_{\Omega}=\rho_{\Omega}^{\dagger}, \rho_{\Omega} \geqslant 0$, and $\operatorname{tr} \rho_{\Omega}=1$. We can appreciate that the solution is not unique since different $\eta(\Omega), \lambda(\Omega)$ can be used at convenience. This freedom may be used, for example, to impose that $\rho_{\Omega}$ should be as close as possible to a pure state.

By inverting Eq. (3.3) expressing $\Delta(\Omega)$ in terms of $\rho_{\Omega}$ it is possible to express the Wigner function for any state $\rho$ as being proportional to the overlap $\operatorname{tr}\left(\rho \rho_{\Omega}\right)$

$$
W(\Omega)=\frac{\mu(\Omega)}{\eta(\Omega)} \operatorname{tr}\left(\rho \rho_{\Omega}\right)-\frac{\lambda(\Omega)}{\eta(\Omega)},
$$

where $\mu(\Omega)=N \lambda(\Omega)+\eta(\Omega) \operatorname{tr} \Delta(\Omega)$. This implies that the Wigner function can be directly determined without using inversion formulas as being proportional to a measurement with statistics given by the overlap $\operatorname{tr}\left(\rho \rho_{\Omega}\right)$.

After Eq. (2.5) a similar relation can be derived for $\mathcal{U}\left(\Omega^{\prime}, \Omega\right)$ involving both the generation and detection of the states $\rho_{\Omega}, \rho_{\Omega^{\prime}}$. In a recent work it has been shown that the very same scheme that serves to detect $\rho_{\Omega}$ also serves to generate it [11]. Therefore, the determination of $\mathcal{U}\left(\Omega^{\prime}, \Omega\right)$ can be regarded as a double measuring scheme.

\section{EXAMPLES}

In what follows we particularize the above analysis to two representatives of the most relevant Wigner functions for finite-dimensional systems: continuous [5-8] and discrete $[9,10]$. Dealing with finite-dimensional systems it can be helpful to regard them as representing an abstract angular momentum $j=(N-1) / 2$, using the eigenvectors $|j, m\rangle$ of the component $j_{z}$ as a suitable orthonormal basis. As an illustrative particular example we will focus always on two modes of the electromagnetic field, where the subspaces with fixed total photon number have finite dimension. In this case, the role of the angular momentum is played by the Stokes operators and the correspondence between the $|j, m\rangle$ basis and the photon-number basis can be expressed as

$$
\left|j=\frac{n_{1}+n_{2}}{2}, m=\frac{n_{1}-n_{2}}{2}\right\rangle=\left|n_{1}=j+m\right\rangle\left|n_{2}=j-m\right\rangle,
$$

where $\left|n_{1}\right\rangle\left|n_{2}\right\rangle$ are number states with $n_{1}$ and $n_{2}$ photons in the corresponding mode.

\section{A. SU(2) Wigner function}

We first consider the operator-function correspondence when the phase space is a sphere so that $\Omega=(\theta, \phi)$, where $\theta$ and $\phi$ are the polar and azimuthal angles, respectively. The most relevant proposals that used this phase space were introduced in Refs. [5,6]. Their equivalence is recalled in the Appendix. For definiteness, we follow the notation in Ref. [6]

$$
\Delta^{j}(\Omega)=\sum_{m, m^{\prime}=-j}^{j} Z_{m, m^{\prime}}^{j}(\Omega)|j, m\rangle\left\langle j, m^{\prime}\right|,
$$

with

$$
\begin{aligned}
Z_{m, m^{\prime}}^{j}(\Omega)= & \frac{\sqrt{4 \pi}}{2 j+1} \sum_{\ell=0}^{2 j} \sqrt{2 \ell+1}\left\langle j, \ell ; m, m^{\prime}-m \mid j, m^{\prime}\right\rangle \\
& \times Y_{\ell, m^{\prime}-m}(\Omega),
\end{aligned}
$$

where $\left\langle j_{1}, j_{2} ; m_{1}, m_{2} \mid j, m\right\rangle$ are the Clebsch-Gordan coefficients and $Y_{\ell, m}(\Omega)$ the spherical harmonics.

By construction there is covariance under $\mathrm{SU}(2)$ transformations $R(\Omega)$

$$
\Delta^{j}(\Omega)=R(\Omega) \Delta^{j}(0) R^{-1}(\Omega) .
$$

For our example of a two mode-field, the SU(2) transformations are all the lossless energy conserving transformations, such as lossless beam splitters and phase plates. The above property allows us to focus on the point $\Omega=0$ without loss of generality. It can be easily seen that $\Delta^{j}(0)$ is diagonal in the $|j, m\rangle$ basis 


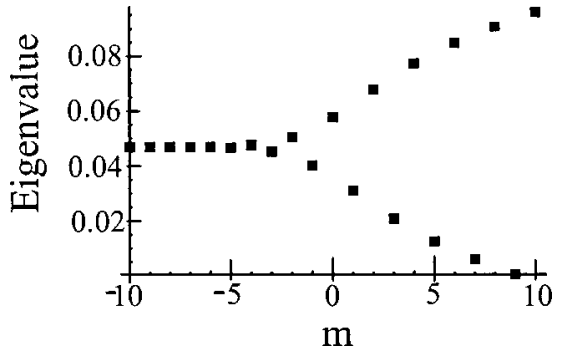

FIG. 1. Eigenvalues of $\rho_{\Omega=0}$ corresponding to the eigenvectors $|j, m\rangle$ as a function of $m$ for $j=10, \eta(0)=1$ and minimum $\lambda(0)$.

$$
\Delta^{j}(0)=\sum_{m=-j}^{j} Z_{m, m}^{j}(0)|j, m\rangle\langle j, m|,
$$

where the eigenvalues $Z_{m, m}^{j}(0)$ are

$$
Z_{m, m}^{j}(0)=\sum_{\ell=0}^{2 j} \frac{2 \ell+1}{2 j+1}\langle j, \ell ; m, 0 \mid j, m\rangle .
$$

For the lowest values of $j$ we have

$$
Z^{1 / 2}(0)=\left(\begin{array}{c}
1.36 \\
-0.36
\end{array}\right), \quad Z^{1}(0)=\left(\begin{array}{c}
1.57 \\
-0.72 \\
0.15
\end{array}\right),
$$

where the component $Z_{j, j}^{j}$ is at the top of the vectors. Using the correspondence (3.3) we see that the phase space point state $\rho_{\Omega=0}$ is also diagonal in the $|j, m\rangle$ basis. In Fig. 1 we have represented the eigenvalues of the eigenvectors $|j, m\rangle$ as a function of $m$ for $\rho_{\Omega=0}$ with $j=10, \eta(0)=1$ and minimum value for $\lambda(0)$. Incidentally, for all the values of $j$ examined we have observed that the larger eigenvalue of $\Delta(\Omega)$ corresponds to the eigenvector $R(\Omega)|j, m=j\rangle$. Accordingly, the pure state with a larger overlap with $\Delta(\Omega)$ would be the $\mathrm{SU}(2)$ coherent state $R(\Omega)|j, m=j\rangle$. This establishes an interesting connection between the Wigner function and the $\mathrm{Q}$ function on the sphere [17].

Focusing on our optical example, a relevant conclusion of these results is that a direct and complete determination of the Wigner function can be carried out simply by photon number detection after a controllable beam splitter. This is because of the correspondence (4.1) between the $j, m$ variables and the number of photons and the implementation of $\mathrm{SU}(2)$ transformations $R(\Omega)$ by beam splitters.

\section{B. Discrete Wigner function}

A continuous phase space is a highly redundant representation for a finite-dimensional space. If we are interested in removing redundancies we can restrict the domain of definition of the above functions to a finite set of points [18]. Maybe a more consistent approach considers a Wigner-Weyl operator-function correspondence formulated from the very beginning on a discrete and finite set of points (which tends to be continuous in the classical limit) $[9,10]$. We focus on the operator-function correspondence introduced in Ref. [10], where the phase space is made of $N \times N$ points as the product of the spectra of the two conjugate variables: $j_{z}$ and azimuthal angle $\phi$. In this case, $\Omega=(m, s)$, with $m, s=-j,-j+1, \ldots, j$, and

$$
\Delta(\Omega)=\frac{1}{2 j+1} \sum_{k, \ell=k_{0}, \ell_{0}}^{k_{0}+2 j, \ell_{0}+2 j} e^{i \gamma_{k, \ell}} e^{-i 2 \pi(k s+\ell m) /(2 j+1)} E^{k} F^{\ell},
$$

where $k, \ell, k_{0}, \ell_{0}$ are integers, $\gamma_{k, \ell}$ constant phases, and $E, F$ are unitary operators

$$
F=e^{i 2 \pi j_{z} /(2 j+1)}, \quad E=e^{i \phi},
$$

where $\phi$ represents here the azimuthal angle operator

$$
\phi=\sum_{s=-j}^{j} \phi_{s}\left|j, \phi_{s}\right\rangle\left\langle j, \phi_{s}\right|
$$

whose eigenvectors and eigenvalues are

$$
\left|j, \phi_{s}\right\rangle=\frac{1}{\sqrt{2 j+1}} \sum_{m=-j}^{j} e^{-i m \phi_{s}}|j, m\rangle, \quad \phi_{s}=\frac{2 \pi}{2 j+1} s .
$$

Unfortunately there is no simple expression for the constant phases $\gamma_{k, \ell}$, which otherwise are not unique [10].

Let us examine in some detail two cases of lower dimensionality $j=1 / 2,1$. For $j=1 / 2$ (dimension two) a possible and simple choice for the parameters is $k_{0}=\ell_{0}=0$ so that $k, \ell=0,1$ and $\gamma_{k, \ell}=\pi k \ell / 2$. The kernel $\Delta(\Omega)$ can be expressed as

$$
\Delta(\Omega)=\frac{1}{2} I_{2}-2 m s \sigma_{x}+s \sigma_{y}+m \sigma_{z}
$$

where $\sigma_{j}$ are the Pauli matrices. Performing the equivalence (3.3) with $\eta(\Omega)=1$ and minimum $\lambda(\Omega)$ we get that the $\rho_{\Omega}$ are the SU(2) coherent states (expressed in the photon number basis $\left.\left|n_{1}\right\rangle\left|n_{2}\right\rangle\right)$

$$
|m, s\rangle=e^{i \phi_{m, s} \sin \frac{\theta_{m, s}}{2}}|0\rangle|1\rangle+\cos \frac{\theta_{m, s}}{2}|1\rangle|0\rangle,
$$

with

$$
\begin{gathered}
\theta_{m, s}=\theta_{0}\left(m+\frac{1}{2}\right)-\left(\pi-\theta_{0}\right)\left(m-\frac{1}{2}\right), \\
\phi_{m, s}=m(1-4 s) \frac{\pi}{2},
\end{gathered}
$$

being $\tan \theta_{0}=\sqrt{2}$. These states can be easily generated and detected since they are the action of a beam splitter on a single photon.

For the case $j=1$ (actually for any odd $j$ ) there is a choice for the phases $\gamma_{k, \ell}$ that allow us to write the phase space point operators in a simple and useful form

$$
\Delta(\Omega)=E^{m} F^{\dagger s} \Delta(0) F^{s} E^{\dagger m},
$$

with 


$$
\Delta(0)=\sum_{m=-j}^{j}|j,-m\rangle\langle j, m| .
$$

As before, let us focus first on the case $\Delta(0)$ and later we will consider the rest of points as a result of the action of the operator $E^{m} F^{\dagger s}$.

In this case, the simplest relation of $\Delta(0)$ with a quantum state is of the form [with $\lambda(0)=-\eta(0)]$

$$
\Delta(0)=I_{3}-2\left|\Omega_{0}\right\rangle\left\langle\Omega_{0}\right|,
$$

where (referring again to the photon number basis $\left|n_{1}\right\rangle\left|n_{2}\right\rangle$ )

$$
\left|\Omega_{0}\right\rangle=\frac{1}{\sqrt{2}}(|2\rangle|0\rangle-|0\rangle|2\rangle) .
$$

This state is rather popular in quantum optics, specially in the area of multiparticle interference [19]. It can be easily generated when two photons impinge simultaneously on the two input ports of a symmetrical beam splitter. The process of detection (i.e., the projection on $\left|\Omega_{0}\right\rangle$ ) can be performed by detecting the presence or absence of coincidences of two photodetectors placed at the outport ports of a symmetrical beam splitter [19].

Finally, until the end of the paper we consider the implementation of the rest of phase space point operators for $\Omega$ $\neq 0$ as the action of powers of $E$ and $F$ on the vector $\left|\Omega_{0}\right\rangle$. The operator $F$ is very simple to implement in quantum optics because $j_{z}=\left(a_{1}^{\dagger} a_{1}-a_{2}^{\dagger} a_{2}\right) / 2$ and $F$ is simply a phase shift. Concerning the action of $E^{m}$ with $m= \pm 1$ we have

$$
\begin{aligned}
& \left|\Omega_{1}\right\rangle=E\left|\Omega_{0}\right\rangle=\frac{1}{\sqrt{2}}(-|1\rangle|1\rangle+|0\rangle|2\rangle), \\
& \left|\Omega_{-1}\right\rangle=E^{-1}\left|\Omega_{0}\right\rangle=\frac{1}{\sqrt{2}}(|1\rangle|1\rangle-|2\rangle|0\rangle) .
\end{aligned}
$$

In order to generate and detect these states we propose a method involving the coupling of the two field modes with an auxiliary two-level system (a two-level atom for instance) expanded by the orthonormal states $| \pm\rangle$. The coupling is of the form

$$
H=-\hbar \eta(|+\rangle\langle+|-|-\rangle\langle-|) \mathbf{a}^{\dagger} \mathcal{U} \mathbf{a}
$$

where

$$
\mathbf{a}=\left(\begin{array}{l}
a_{1} \\
a_{2}
\end{array}\right), \quad \mathcal{U}=\left(\begin{array}{cc}
\gamma_{0}+\gamma_{3}, & \gamma_{1}-i \gamma_{2} \\
\gamma_{1}+i \gamma_{2}, & \gamma_{0}-\gamma_{3}
\end{array}\right),
$$

being $\eta$ and $\gamma_{j}, j=0, \ldots, 3$, constants. This coupling represents a beam splitter controlled by the state of the auxiliary system, and can be easily achieved in practice via the nonresonant interaction of the field modes with a two-level atom. A suitable initial state for the two-level system is

$$
\left|\varphi_{+}\right\rangle=\frac{1}{\sqrt{2}}(|+\rangle+|-\rangle) .
$$

After an interaction time $\tau$ such that $\eta \tau=1$ we get

$$
e^{-i \tau H / \hbar}|1\rangle|1\rangle\left|\varphi_{+}\right\rangle=\frac{1}{\sqrt{2}}\left(e^{2 i \gamma_{0}}\left|\psi_{+}\right\rangle|+\rangle+e^{-2 i \gamma_{0}}\left|\psi_{-}\right\rangle|-\rangle\right),
$$

where, in the photon number basis,

$$
\begin{gathered}
\left|\psi_{+}\right\rangle=\sqrt{2} r t|2\rangle|0\rangle-\sqrt{2} r^{*} t^{*}|0\rangle|2\rangle+\left(|t|^{2}-|r|^{2}\right)|1\rangle|1\rangle, \\
\left|\psi_{-}\right\rangle=-\sqrt{2} r t^{*}|2\rangle|0\rangle+\sqrt{2} r^{*} t|0\rangle|2\rangle+\left(|t|^{2}-|r|^{2}\right)|1\rangle|1\rangle,
\end{gathered}
$$

being

$$
t=\cos \gamma+i \frac{\gamma_{3}}{\gamma} \sin \gamma, \quad r=\frac{\gamma_{2}+i \gamma_{1}}{\gamma} \sin \gamma,
$$

and $\gamma=\sqrt{\gamma_{1}^{2}+\gamma_{2}^{2}+\gamma_{3}^{2}}$ [20]. The target states (4.19) are generated by detecting whether the output auxiliary system is in the states

$$
\left|\varphi_{ \pm}\right\rangle=\frac{1}{\sqrt{2}}(|+\rangle \pm|-\rangle)
$$

provided that the coupling parameters verify that $\gamma_{0}=3 \pi / 8$, $\arg (t)=3 \pi / 4, \arg (r)=\pi / 2,|t|=\cos \vartheta$, and $|r|=\sin \vartheta$, with $\vartheta=\pi / 8$. In such a case

$$
\left\langle\varphi_{ \pm}\left|e^{-i \tau H / \hbar}\right| 1\right\rangle|1\rangle\left|\varphi_{+}\right\rangle=\frac{1}{2}\left(e^{2 i \gamma_{0}}\left|\psi_{+}\right\rangle \pm e^{-2 i \gamma_{0}}\left|\psi_{-}\right\rangle\right) \propto\left|\Omega_{ \pm 1}\right\rangle .
$$

This shows how the states $\left|\Omega_{ \pm 1}\right\rangle$ can be generated. Concerning their detection (i.e., the measurement of the projection on $\left|\Omega_{ \pm 1}\right\rangle$ ) the same procedure above leads us to

$$
\left\langle\varphi_{ \pm}\right|\langle 1|\left\langle 1\left|e^{i \tau H / \hbar}\right| \varphi_{+}\right\rangle \propto\left\langle\Omega_{ \pm 1}\right| .
$$

Therefore, we have to couple the two-mode field to the auxiliary system prepared in the state $\left|\varphi_{+}\right\rangle$and the coupling must be given by the replacement of $\gamma_{j} \rightarrow-\gamma_{j}$ in Eqs. (4.20) and (4.21). After the coupling we perform the $\left|\varphi_{ \pm}\right\rangle$measurement on the auxiliary system and a joint photon detection in the field modes.

This completes the practical procedure to measure the discrete Wigner function for $j=1$ and to generate and detect the corresponding phase space point states.

\section{CONCLUSIONS}

We have shown that for finite-dimensional spaces there are quantum states that can be regarded as suitable counterparts of the points of the classical phase space. Theses states define a procedure to measure the Wigner function at each point of the phase space independently. This applies both to the Wigner functions of states and transformations. We have illustrated these ideas applying them to two definitions of the finite-dimensional Wigner functions on different phase spaces: discrete and continuous. 


\section{APPENDIX: SU(2) WIGNER FUNCTION EQUIVALENCE}

Here we show that the SU(2) Wigner function introduced in Ref. [5] coincides with the approach introduced in Ref. [6]. Further equivalences between Wigner functions can be found in Ref. [8].

The phase space point operators $\Delta(\Omega)$ are introduced in Ref. [5] as

$$
\begin{aligned}
\Delta(\Omega)= & \sum_{m, m^{\prime}=-j}^{j} \sum_{k=0}^{2 j} \sum_{q=-k}^{k}(-1)^{j-m} \sqrt{2 k+1}\left(\begin{array}{lll}
j & k & j \\
-m & q & m^{\prime}
\end{array}\right) \\
& \times Y_{k, q}(\Omega)\left|j, m^{\prime}\right\rangle\langle j, m|,
\end{aligned}
$$

where the term in parentheses is a $3 j$ symbol. The desired equivalence is proved once we notice that

$$
\begin{aligned}
\left(\begin{array}{lll}
j & k & j \\
-m & q & m^{\prime}
\end{array}\right) & =(-1)^{2 j+k}\left(\begin{array}{lll}
j & k & j \\
m^{\prime} & q & -m
\end{array}\right) \\
& =\frac{(-1)^{3 j+m}}{\sqrt{2 j+1}}\left\langle j, k ; m^{\prime}, q \mid j, m\right\rangle .
\end{aligned}
$$

Note that Refs. [5] and [6] use a slightly different definition for $d \Omega$,

$$
d \Omega=\sqrt{\frac{2 j+1}{4 \pi}} \sin \theta d \theta d \phi, \quad d \Omega=\frac{2 j+1}{4 \pi} \sin \theta d \theta d \phi,
$$

respectively.
[1] U. Leonhardt and H. Paul, Prog. Quantum Electron. 19, 89 (1995); U. Leonhardt, Measuring the Quantum State of Light (Cambridge University Press, Cambridge, England, 1997); D.-G. Welsch, W. Vogel, and T. Opatrný, in Progress in Optics, edited by E. Wolf (Elsevier Science, Amsterdam, 1999), Vol. 39.

[2] J. F. Poyatos, J. I. Cirac, and P. Zoller, Phys. Rev. Lett. 78, 390 (1997); I. L. Chuang and M. A. Nielsen, J. Mod. Opt. 44, 2455 (1997); G. M. D’Ariano and L. Maccone, Phys. Rev. Lett. 80, 5465 (1998); V. Bužek, Phys. Rev. A 58, 1723 (1998); M. A. Nielsen, E. Knill, and R. Laflamme, Nature (London) 396, 52 (1998); R. Gutzeit, S. Wallentowitz, and W. Vogel, Phys. Rev. A 61, 062105 (2000); A. M. Childs, I. L. Chuang, and D. W. Leung, ibid. 64, 012314 (2001); J. Fiurášek and Z. Hradil, ibid. 63, 020101(R) (2001); J. Fiurášek, ibid. 64, 024102 (2001); G. M. D’Ariano and P. Lo Presti, Phys. Rev. Lett. 86, 4195 (2001); quant-ph/0211133; F. De Martini, A. Mazzei, M. Ricci, and G. M. D'Ariano, quant-ph/0207143; quant-ph/ 0210210; J. B. Altepeter, D. Branning, E. Jeffrey, T. C. Wei, P. G. Kwiat, R. T. Thew, J. L. O'Brien, M. A. Nielsen, and A. G. White, Phys. Rev. Lett. 90, 193601 (2003).

[3] H. Groenewold, Physica (Utrecht) 12, 405 (1946); K. E. Cahill and R. J. Glauber, Phys. Rev. 177, 1857 (1969); 177, 1882 (1969); M. Hillery, R. F. O'Connell, M. O. Scully, and E. P. Wigner, Phys. Rep. 106, 121 (1984).

[4] N. Mukunda, Am. J. Phys. 47, 182 (1979); J. P. Bizarro, Phys. Rev. A 49, 3255 (1994); J. Vaccaro, ibid. 52, 3474 (1995); Opt. Commun. 113, 421 (1995); P. Kasperkovitz and M. Peev, Ann. Phys. (N.Y.) 230, 21 (1994).

[5] G. S. Agarwal, Phys. Rev. A 24, 2889 (1981); J. P. Dowling, G. S. Agarwal, and W. P. Schleich, ibid. 49, 4101 (1994).

[6] J. C. Várilly and J. M. Gracia-Bondía, Ann. Phys. (N.Y.) 190, 107 (1989).

[7] D. M. Kaplan and G. C. Summerfield, Phys. Rev. 187, 639 (1969); M. O. Scully, Phys. Rev. D 28, 2477 (1983); G. Ramachandran, A. R. Usha-Devi, P. Devi, and S. Sirsi, Found. Phys. 26, 401 (1996); S. M. Chumakov, A. Frank, and K. B. Wolf, Phys. Rev. A 60, 1817 (1999); C. Brif and A. Mann, ibid. 59, 971 (1999); V. P. Karassiov and A. V. Masalov, J. Opt. B: Quantum Semiclassical Opt. 4, S366 (2002).

[8] S. M. Chumakov, A. B. Klimov, and K. B. Wolf, Phys. Rev. A 61, 034101 (2001).

[9] W. K. Wootters, Ann. Phys. (N.Y.) 176, 1 (1987); O. Cohendet, Ph. Combe, M. Sirugue, and M. Sirugue-Collin, J. Phys. A 21, 2875 (1988); D. Galetti and A. F. R. Toledo Piza, Physica A 149, 267 (1988); J. A. Vaccaro and D. T. Pegg, Phys. Rev. A 41, 5156 (1990); A. Lukš and V. Peřinová, Quantum Opt. 6, 125 (1994); U. Leonhardt, Phys. Rev. Lett. 74, 4101 (1995); Phys. Rev. A 53, 2998 (1996); T. Opatrný, D.-G. Welsch, and V. Bužek, ibid. 53, 3822 (1996); C. Miquel, J. P. Paz, and M. Saraceno, ibid. 65, 062309 (2002); J. P. Paz, ibid. 65, 062311 (2002).

[10] A. Luis and J. Peřina, J. Phys. A 31, 1423 (1998).

[11] A. Luis and L. L. Sánchez-Soto, Phys. Lett. A 261, 12 (1999).

[12] A. Luis and L. L. Sánchez-Soto, Phys. Rev. Lett. 83, 3573 (1999); A. Luis, Phys. Rev. A 62, 054302 (2000).

[13] L. G. Lutterbach and L. Davidovich, Phys. Rev. Lett. 78, 2547 (1997); G. Nogues, A. Rauschenbeutel, S. Osnaghi, P. Bertet, M. Brune, J. M. Raimond, S. Haroche, L. G. Lutterbach, and L. Davidovich, Phys. Rev. A 62, 054101 (2000); P. Bertet, A. Auffeves, P. Maioli, S. Osnaghi, T. Meunier, M. Brune, J. M. Raimond, and S. Haroche, Phys. Rev. Lett. 89, 200402 (2002); R. Juárez-Amaro and H. Moya-Cessa, Phys. Rev. A 68, 023802 (2003).

[14] U. Leonhardt and H. Paul, Phys. Lett. A 193, 117 (1994).

[15] A. Luis (unpublished).

[16] A. J. Bracken, J. Phys. A 36, L329 (2003).

[17] F. T. Arecchi, E. Courtens, R. Gilmore, and H. Thomas, Phys. Rev. A 6, 2211 (1972).

[18] J.-P. Amiet and S. Weigert, J. Opt. B: Quantum Semiclassical Opt. 2, 118 (2000).

[19] C. K. Hong, Z. Y. Ou, and L. Mandel, Phys. Rev. Lett. 59, 2044 (1987); A. M. Steinberg, P. G. Kwiat, and R. Y. Chiao, ibid. 68, 2421 (1992).

[20] A. Luis and L. L. Sánchez-Soto, Quantum Semiclassic. Opt. 7, 153 (1995). 